\title{
The Streptococcus milleri group in chronic obstructive pulmonary disease
}

\author{
Lucie Navratilovaa , Jan Bardon ${ }^{\mathrm{b}}$, Radko Novotnya, Jaromir Zatloukal', Petr Jakubec', Vitezslav Kolekc, Martin Zapalkad,

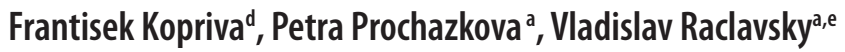

\begin{abstract}
Background and Aims. S. anginosus, constellatus and intermedius, also known as the Streptococcus milleri group (SMG) are three streptococcal species more frequently detected in cases of invasive disease, abscesses and empyema in particular. Recent research suggests they play a role in exacerbations of cystic fibrosis (CF). Owing to poor recovery on standard culture media and difficult differentiation from non-pathogenic streptococci, SMG may be underdiagnosed in routine settings. We aimed to establish the incidence of SMG in chronic obstructive pulmonary disease (COPD) patients compared to CF patients and to examine possible links of SMG to exacerbations that plays a key role in progression of COPD.

Methods. Altogether, 90 respiratory tract samples of patients suffering from CF or COPD were examined during the period from July 2012 to December 2013. Semi-selective McKay agar was used for primary cultivation of SMG and MALDI TOF MS was used for species identification that was confirmed by biochemical profiling and specific PCR.

Results. We confirmed the presence of SMG in CF (17.6\% incidence in adult patients) and newly established its presence in COPD (10.3\% incidence). In COPD, SMG was detected in 4 cases of acute exacerbations, where no other bacterial pathogen was detected. In 3/4 cases, increased CRP level indicated bacterial infection as a cause of the exacerbation and in all 3 cases, patients recovered during antibiotic treatment.
\end{abstract}

Conclusions. Our data indicate SMG may act as opportunist pathogens able to cause exacerbations in COPD.

Key words: Streptococcus milleri group, Streptococcus anginosus, Streptococcus constellatus, Streptococcus intermedius, cystic fibrosis, chronic obstructive pulmonary disease

Received: November 20, 2015; Accepted with revision: March 18, 2016; Available online: April 21, 2016 http://dx.doi.org/10.5507/bp.2016.017

\author{
${ }^{a}$ Department of Microbiology, Faculty of Medicine and Dentistry, Palacky University Olomouc, Czech Republic \\ 'State Veterinary Institute in Olomouc, Jakoubka ze Stribra 1, Olomouc, Czech Republic \\ 'Department of Respiratory Medicine, University Hospital Olomouc, Czech Republic \\ ${ }^{d}$ Department of Pediatrics, University Hospital Olomouc, Czech Republic \\ eInstitute of Molecular and Translational Medicine, Faculty of Medicine and Dentistry, Palacky University Olomouc, Czech Republic \\ Corresponding author:Vladislav Raclavsky, e-mail: vladislav.raclavsky@upol.cz
}

\section{INTRODUCTION}

The Streptococcus milleri group (SMG) consists of three streptococcal species - S. anginosus, constellatus and intermedius - frequently detected in cases of invasive disease, abscesses and empyema in particular ${ }^{1}$. SMG has also been suggested to play role in pneumonia and other lower respiratory tract infections ${ }^{2}$, mainly in cases of empyema and lung abscesses ${ }^{3.5}$. Some authors were able to document cases where pleuropulmonary SMG infection followed pneumonia ${ }^{6,7}$. Recently, the role of SMG in exacerbations of pulmonary disease in cystic fibrosis (CF) patients has been suggested by a Canadian group ${ }^{8,9}$. Unfortunately, recovery of SMG from clinical samples is hindered by the fact that some isolates do not grow well on traditional laboratory media or can be dismissed as clinically irrelevant normal microbiota. Recovery rate is further decreased in non-sterile samples such as sputum due to growth competition between different bacterial species. This may explain why SMG has not been confirmed as a significant pathogen in cystic fibrosis by other groups to date, with the exception of one case report ${ }^{10}$.
Shortcomings of the traditional media in recovery of SMG can be overcome by the use of semi-selective McKay $\operatorname{agar}^{11}$ or NAS agar ${ }^{12}$. Successful differentiation of SMG from other streptococcal species can also be difficult but can be overcome using the MALDI TOF MS technology. However, even if combining semi-selective cultivation and MALDI TOF MS, recovery of SMG can be delayed because of the slow growth of these bacteria. In an attempt to facilitate their detection in clinical sample, three PCR strategies were developed independently ${ }^{13-15}$. In our study, patients suffering two chronic lung diseases were recruited to provide samples for the study. CF patients, where the presence and role of SMG was already established, were recruited to provide samples for evaluation of available strategies for SMG detection in terms of simple and economic performance in routine settings. Chronic obstructive pulmonary disease (COPD) patients were recruited to establish the incidence of SMG in COPD and examine possible links of SMG to exacerbations that play key role in progression of this chronic disease. 


\section{METHODS}

\section{Bacterial strains and cultivation}

The study was approved by the Institutional Review Board and all patients enrolled signed the informed consent form. Samples from respiratory tract (sputum, nasopharyngeal swab) of patients suffering chronic lung disease were examined for the presence of SMG during the period from July 2012 to December 2013. Most of the samples (>71\%) were collected either during stable disease or at the onset of exacerbation preceding the start of antibiotic therapy. Sputum samples from CF and COPD patients were liquefied by adding roughly equal volumes of $2 \%$ $\mathrm{N}$-acetylcysteine solution and homogenised by shaking with glass beads, diluted and inoculated on McKay agar (prepared by Oxoid for this study) which was developed earlier to selectively follow the SMG population ${ }^{9,11}$. For economic reasons, $10^{5}$-times dilution was plated on one half of McKay plate to detect growth of bacterial concentrations $\geq 10^{6} \mathrm{CFU} / \mathrm{mL}$ ( 10 colonies or more), and $10^{7}$-dilution was plated on another half of the same plate to facilitate differentiation of colonies if bacterial concentration was higher the $10^{7} \mathrm{CFU} / \mathrm{mL}$. If sputum was not available in younger patients, pharyngeal swabs were taken and inoculated on McKay agar. Altogether, $21 \mathrm{CF}$ patients from the Dept. of Pediatrics, 19 CF patients from the Dept. of Respiratory Medicine and 57 COPD patients from the same department, University Hospital Olomouc, were enrolled in the study. All colonial types that were recovered reliably on McKay agar were subcultured on Columbia blood agar (CBA) and MALDI TOF MS was used as primary tool for species identification as described below. Only isolates identified as SMG by MALDI TOF MS were further tested. If the same species was recovered from the same patient at different time points, all isolates were further tested. Conventional biochemical profiling and species-specific PCR ( ref. $^{13}$ ) were used to confirm the primary identification achieved by MALDI TOF MS, as described below.

\section{MALDI TOF MS}

Bacterial isolates were subcultured on CBA and toothpick sampling was used to prepare a direct smear on MALDI TOF target plate, which was then overlaid by $1 \mu \mathrm{L}$ of $70 \%$ formic, air dried and overlaid by $1 \mu \mathrm{L}$ of matrix solution ( $\alpha$-cyano-4-hydroxycinnamic acid solution; HCCA, Sigma-Aldrich). A MALDI Microflex LT instrument (Bruker) was used for identification in automated mode in accordance with manufacturers' instructions (MALDI Biotyper 3.1 User Manual Revision 1). If the confidence score values dropped below 2.0, usertargeted laser shots were performed to improve the score. Conventional biochemical profiling, Lancefield classification and sequence-specific PCR were performed for best avoidance of any misidentifications (see below).

\section{Biochemical profiling and Lancefield classification}

The RapID STR System (Remel, Oxoid) was used for conventional biochemical identification, and the
PathoDxtra Strep grouping kit (Thermo Scientific) was used to determine the Lancefield group of SMG isolates, both in accordance with manufacturer's instructions.

\section{Hyaluronidase activity}

Hyaluronidase activity was detected using the decapsulation test as described earlier ${ }^{16}$. Briefly, CBA was inoculated by a broad line of Streptococcus equi producing a hyaluronic acid capsule. SMG isolates were inoculated as a thin line perpendicular to the broad $S$. equi line with 1-2 mm penetration into the broad $S$. equi line. Plates were incubated for $24 \mathrm{~h}$ at $37{ }^{\circ} \mathrm{C}$ with $5 \% \mathrm{CO}_{2}$ in a humid chamber. After incubation, the test was evaluated as positive if discernible suppression of mucosal growth of $S$. equi was observed in a semi-circular zone around the point of SMG line penetration into the $S$. equi line.

\section{Haemolytic activity and capsule formation}

Haemolysis was evaluated after prolonged cultivation (72-96 h) under anaerobic conditions $\left(80 \% \mathrm{~N}_{2}, 10 \% \mathrm{H}_{2}\right.$, $10 \% \mathrm{CO}_{2}$ ) on Müller-Hinton ( $\left.\mathrm{MH}\right)$ agar supplemented with 7\% horse blood (Trios, Czech Republic) to increase the sensitivity of detection of haemolytic activity. Capsule formation was evaluated after $48 \mathrm{~h}$ cultivation on $\mathrm{BHI}$ agar (Trios, Czech Republic) under anaerobic conditions $\left(80 \% \mathrm{~N}_{2}, 10 \% \mathrm{H}_{2}\right.$, and $\left.10 \% \mathrm{CO}_{2}\right)$. The capsule was visualized using Burri's India ink technique with carbol fuchsin for counterstaining.

\section{Penicillin susceptibility testing}

Isolated colonies from 24-hour aerobic cultures on CBA were suspended in sterile Mueller-Hinton broth to a $0.5 \mathrm{McFarland}$ standard. $\mathrm{MH}$ agar plates supplemented with 7\% horse blood (Trios, Czech Republic) were inoculated by pouring suspended bacterial culture and E-test strips (benzylpenicillin PGL 0.002-32 $\mu \mathrm{g} / \mathrm{mL}$, bioMèrieux) were placed on the plates to determine MIC of penicillin. The clinical breakpoint recommended by the European Committee on Antimicrobial Susceptibility Testing (EUCAST, www.eucast.org) for susceptible viridans group streptococci $(\leq 0.25 \mu \mathrm{g} / \mathrm{mL})$ was used.

\section{DNA extraction}

Isolates were grown on CBA for $24 \mathrm{~h}$ as described above and 1-3 colonies were suspended in $50 \mu \mathrm{L}$ of deionised water and lysed by heating for $10 \mathrm{~min}$ at $94{ }^{\circ} \mathrm{C}$. Crude lysates were spun down $(13000 \times \mathrm{g}, 2 \mathrm{~min})$ and the supernatant was transferred to a new tube and stored at $-20{ }^{\circ} \mathrm{C}$ until used.

\section{PCR}

Two previously published protocols were used - one for one-round real-time PCR (ref. ${ }^{14}$ ) and the other one for two-round nested PCR (ref. ${ }^{13}$ ). List of primer sets targeting $16 \mathrm{~S}$ rDNA is given in Table 1 . All the $15 \mu \mathrm{L}$ PCR reactions were performed with $7.5 \mu \mathrm{L}$ Lightcycler 480 HRM master mix, $4.4 \mu \mathrm{L}$ HRM water (PCR-grade), 0.9 $\mu \mathrm{L} \mathrm{MgCl}{ }_{2}$ (final concentration $1.5 \mathrm{mmol} / \mathrm{L}$ ), $0.1 \mu \mathrm{L}$ of each forward and reverse primers (Table 1) and $2 \mu \mathrm{L}$ of DNA template. PCR amplification was performed in a 
Table 1. List of primers.

\begin{tabular}{|c|c|c|c|c|c|}
\hline Species & Name of primer & Sequence $\left(5^{\prime} \rightarrow 3^{\prime}\right)$ & $\begin{array}{l}\text { Size } \\
(\mathrm{bp})\end{array}$ & Type of PCR & Ref. \\
\hline \multirow{2}{*}{ S. anginosus } & 16S_SA_F & CAAGTAGGACGCACAGTTTA & \multirow{2}{*}{146} & \multirow{4}{*}{ Real-time PCR } & \multirow{4}{*}{14} \\
\hline & 16S_SA_R & TGTGTTACATACTGTTATGCGGT & & & \\
\hline \multirow{2}{*}{$\begin{array}{l}\text { S. constellatus/ } \\
\text { S. intermedius }\end{array}$} & 16S_SCI_F & AAGTAGAACGCACAGGATG & \multirow{2}{*}{143} & & \\
\hline & 16S_SCI_R & CAGTAAATGTTCTTATGCGGTATTAG & & & \\
\hline \multirow{2}{*}{ SMG } & $27 \mathrm{~F}$ & AGAGTTTGATCMTGGCTCAG & \multirow{2}{*}{1505} & \multirow{2}{*}{$\begin{array}{l}\text { Nested PCR } \\
-1 \text { st round }\end{array}$} & \multirow{8}{*}{13} \\
\hline & $1492 \mathrm{R}$ & TACGGYTACCTTGTTACGACTT & & & \\
\hline \multirow{2}{*}{ S. anginosus } & SA_F & ATGCAATTGCATCGCTAGT & \multirow{2}{*}{445} & \multirow{6}{*}{$\begin{array}{l}\text { Nested PCR } \\
-2 \text { nd round }\end{array}$} & \\
\hline & SA_R & GCAGGCTTTGGAAACTGTTTAACT & & & \\
\hline \multirow{2}{*}{ S. constellatus } & SC_F & GTGCAAGAGCATCACTACC & \multirow{2}{*}{445} & & \\
\hline & SC_R & GCAGGCTTTGGAAACTGTTTAACT & & & \\
\hline \multirow{2}{*}{ S. intermedius } & SI_F & GTGCAAATGCATCACTACC & \multirow{2}{*}{445} & & \\
\hline & SI_R & GCAGGCTTTGGAAACTGTTTAACT & & & \\
\hline
\end{tabular}

CFX96 Real-time system thermocycler (Bio-Rad). In the real time PCR protocol, primers 16S_SA_F, 16S_SA_R for S. anginosus and 16S_SCI_F and 16S_SCI_R for $S$. constellatus/intermedius were used with initial denaturation at $95{ }^{\circ} \mathrm{C}$ for $10 \mathrm{~min}$, followed by 30 cycles of denaturation at $94{ }^{\circ} \mathrm{C}$ for $10 \mathrm{~s}$, annealing at $61{ }^{\circ} \mathrm{C}$ for $15 \mathrm{~s}$ and extension at $72{ }^{\circ} \mathrm{C}$ for $15 \mathrm{~s}$. In the nested PCR protocol, universal primers $27 \mathrm{~F}$ and $1492 \mathrm{R}$ were used in the first round with initial denaturation at $95{ }^{\circ} \mathrm{C}$ for $10 \mathrm{~min}$, followed by 35 cycles of denaturation at $94^{\circ} \mathrm{C}$ for $60 \mathrm{~s}$, annealing at $55^{\circ} \mathrm{C}$ for $60 \mathrm{~s}$, extension at $72{ }^{\circ} \mathrm{C}$ for $90 \mathrm{~s}$, followed by final extension at $72{ }^{\circ} \mathrm{C}$ for $10 \mathrm{~min}$. The size of first-round predicted PCR product was $1505 \mathrm{bp}$ and $2 \mu \mathrm{L}$ of the first-round PCR mix were used as template in the second-round PCR. Primers SA_F and SA_R for $S$. anginosus, SC_F and SC_R for S. constellatus and SI_F and SI_R for $S$. intermedius were used in the second-round with initial denaturation at $95{ }^{\circ} \mathrm{C}$ for $10 \mathrm{~min}$, followed by 35 cycles of denaturation at $94{ }^{\circ} \mathrm{C}$ for $30 \mathrm{~s}$, annealing at $58.5^{\circ} \mathrm{C}$ for $30 \mathrm{~s}$, and extension at $72{ }^{\circ} \mathrm{C}$ for $30 \mathrm{~s}$. In both the real-time protocol and the nested PCR protocol, melting analysis was performed immediately after PCR amplification as follows: temperature of samples was adjusted to $70{ }^{\circ} \mathrm{C}$ and then heated to $95^{\circ} \mathrm{C}$ with raising by $0.1{ }^{\circ} \mathrm{C}$ per $0.05 \mathrm{~s}$ each step.

\section{RESULTS}

Altogether, 183 sputum samples ( 96 from CF patients and 87 from COPD patients) and 50 pharyngeal swabs (all from younger CF patients) yielded $25 \mathrm{SMG}$ isolates, as identified by MALDI TOF MS. Of these, 16 isolates were from 12 patients suffering from $\mathrm{CF}$ and 9 were from 6 patients suffering from COPD. Then, according to diagnosis, $\mathrm{SMG}$ were recovered from $11.0 \%$ of samples from CF patients, and $10.3 \%$ of samples from COPD patients. Of all the $25 \mathrm{SMG}$ isolates, 18 were $S$. anginosus, 4 were $S$. constellatus and 3 were $S$. intermedius. For the complete list of all species recovered on McKay agar
Table 2. Abbreviated list of species (frequency $\geq 1 \%$ ) recovered on McKay agar.

\begin{tabular}{lc}
\hline Species & $\begin{array}{c}\text { No. }(\%) \\
\text { of times isolated } \\
(\mathrm{n}=670)\end{array}$ \\
\hline All streptococcal species & $307(45.8)$ \\
All non-streptococcal species & $363(54.2)$ \\
Streptococcus mitis group & $98(15.7)$ \\
Streptococcus salivarius & $90(13.4)$ \\
Pseudomonas aeruginosa & $70(10.4)$ \\
Staphylococcus aureus & $49(7.3)$ \\
Streptococcus parasanguinis & $34(5.1)$ \\
Streptococcus milleri group & $25(3.7)$ \\
$\quad$ S. anginosus & $18(2.7)$ \\
$\quad$ S. constellatus & $4(0.6)$ \\
S. intermedius & $3(0.4)$ \\
Stenotrophomonas maltophilia & $20(3.0)$ \\
Rothia mucilaginosa & $18(2.7)$ \\
Streptococcus sanguinis & $15(2.2)$ \\
Burkholderia cenocepacia & $15(2.2)$ \\
Corynebacterium pseudodiphtheriticum & $11(1.6)$ \\
Streptococcus gordonii & $11(1.6)$ \\
Pseudomonas grimontii & $10(1.5)$ \\
Lactobacillus rhamnosus & $9(1.3)$ \\
Streptococcus peroris & $9(1.3)$ \\
Gemella haemolysans & $8(1.2)$ \\
Klebsiella oxytoca & $8(1.2)$ \\
Streptococcus agalactiae & $8(1.2)$ \\
Moraxella catarrhalis & $7(1.0)$ \\
\hline
\end{tabular}

see Table 2. All of our SMG isolates were susceptible to penicillin.

\section{Phenotypic testing}

To confirm the MALDI TOF MS species identification, all the isolates identified as SMG were also identified by conventional biochemical profiling using the RapID STR System (Remel, Oxoid). In all isolates, conventional 
Table 3. Summary of phenotypic characteristics.

\begin{tabular}{lccc}
\hline & $\begin{array}{c}\text { S. anginosus } \\
\%(\mathrm{n}=18)\end{array}$ & $\begin{array}{c}\text { S. constellatus } \\
\%(\mathrm{n}=4)\end{array}$ & $\begin{array}{c}\text { S. intermedius } \\
\%(\mathrm{n}=3)\end{array}$ \\
\hline Hyaluronidase activity & 0 & $100(4)$ & $100(3)$ \\
Beta galactosidase activity & 0 & 0 & $100(3)$ \\
Capsule & $44(8)$ & $100(4)$ & $100(3)$ \\
Alpha haemolysis & $39(7)$ & $50(2)$ & $67(2)$ \\
Beta haemolysis & $11(2)$ & $25(1)$ & $33(1)$ \\
Gamma haemolysis & $50(9)$ & $25(1)$ & 0 \\
Lancefield group C & $33(6)$ & 0 & 0 \\
Lancefield group F & $61(11)$ & $25(1)$ & 0 \\
Lancefield group other & 0 & 0 & 0 \\
Non-typeable & $6(1)$ & $75(3)$ & $100(3)$ \\
\hline
\end{tabular}

biochemical profiles were in accordance with results of MALDI TOF MS identification. Summary of other phenotypic characteristics including Lancefield grouping is given in Table 3.

\section{Genotypic testing}

SMG isolates were reliably distinguished from other species of streptococci and other bacterial species as well. Altogether, 35 isolates of other streptococcal species and 16 isolates of other bacterial species frequently recovered from CF patients (Pseudomonas aeruginosa, Burkholderia cepacia complex, Klebsiella pneumoniae and Escherichia coli) were tested. When using the real-time PCR system ${ }^{14}$, SMG streptococci typically showed crossing points between cycle 10 and 15 , in a few cases latest in cycle 25 , whereas other streptococcal species ( $S$. agalactiae, S. gordonii, S. mutans, S. oralis, S. parasanguinis, S. pneumoniae, $S$. pyogenes, $S$. salivarius and $S$. sanguinis) after more than 30 amplification cycles only. No DNA of other nonstreptococcal species gave positive amplification result. Melting curves of the whole PCR product mix without using probes were identical for all three SMG species and showed a melting temperature $\left(\mathrm{T}_{\mathrm{m}}\right)$ of $81.58 \pm 0.25^{\circ} \mathrm{C}$. When using the nested PCR assay ${ }^{13}$, positive amplification with the first-round primers was observed with all species tested but only SMG were amplified in the second-round reaction. After the second round, melting curves were the same for all three species of SMG and showed $\mathrm{T}_{\mathrm{m}}$ of 86.18 $\pm 0.25^{\circ} \mathrm{C}$. The sensitivity of both assays was compared using diluted DNA template for each SMG species separately, testing was performed in duplicates. As expected, the nested approach was more sensitive, namely $10 \times$ in $S$. anginosus and S. intermedius, and $100 \times$ in S. constellatus.

\section{CF patients}

In this study, SMG streptococci were recovered from $11.0 \%$ of samples from CF patients. However, they were much more frequent in adult CF patients (17.6\% of samples, $n=13$ ) compared to young patients ( $<18$ years, $2.4 \%$ of samples, $n=3$ ). In addition, young patients showed no symptoms of exacerbation at the time of SMG recovery. Then, most probably, SMG found in the respiratory pathways of young CF patients represents colonisation. Therefore, the characteristics of adult-only cases positive for SMG are summarised in Table 4, to avoid bias due to age extremes and low number of cases in younger patients. In our group of 13 adult CF patients positive for SMG, 9 showed symptoms of exacerbation (69\%, 3 inpatients, 6 outpatients). P. aeruginosa was always detected in the exacerbated cases.

\section{COPD patients}

SMG streptococci were recovered from $10.3 \%(n=10)$ of all COPD cases $(n=90)$; altogether, 7 of them showed symptoms of exacerbation (70\%, 5 inpatients, 2 outpatients). This frequency of exacerbations, unfortunately, did not differ from the average frequency of exacerbations in our group of COPD patients (61 exacerbations in 90 cases, i.e. $67.8 \%$ ). Furthermore, SMG streptococci were recovered from $11.5 \%$ cases without exacerbation $(3 / 29)$ compared to $10.3 \%$ cases with acute exacerbation (7/61). For comparison, $H$. influenzae, a well-established opportunist respiratory pathogen in COPD, has been recovered in $20.7 \%$ cases without exacerbation $(6 / 29)$ compared to $19.7 \%$ cases with acute exacerbation (12/61). Moreover, Klebsiella pneumoniae, a well-established primary respiratory pathogen, was recovered in $6.9 \%$ cases without exacerbation $(2 / 29)$ compared to $18 \%$ cases with acute exacerbation (11/61), which was not significant $(P=0.21$, Fisher's exact test). Therefore, we focused on cases where SMG presence and exacerbation occurred concurrently, in detail.

Of the 7 cases of SMG and exacerbation, other bacterial pathogens were detected in 3 cases, namely Moraxella catarrhalis in one case, and Pseudomonas aeruginosa together with Haemophilus influenzae in the other two cases. In 4 of the exacerbated cases (all inpatients) no other bacterial pathogen was detected at the time of SMG recovery. MALDI-TOF species identification was performed once a week at a remote location; therefore, SMG findings were reported to the clinician retrospectively only. Then, the exacerbations were initially treated empirically; this treatment was possibly modified based on reports from routine microbiological examination received typically 48 hours after sampling.

In Case 1, a 72-year-old man suffering from stage 4 COPD was first treated empirically as an outpatient by clarithromycin. Because of the progression, he was admitted to hospital and treated by intravenous ceftazidim 
Table 4. Summary of adult cases positive for SMG.

\begin{tabular}{lcclccl}
\hline $\begin{array}{c}\text { Patient } \\
\text { code }\end{array}$ & Diagnosis & $\begin{array}{c}\text { Date } \\
\text { of recovery }\end{array}$ & \multicolumn{1}{c}{ Species } & $\begin{array}{c}\text { Oupatient/ } \\
\text { Inpatient }\end{array}$ & Exacerbation & \multicolumn{1}{c}{ Other pathogens } \\
\hline ONDRE & CF & 26.06 .2012 & S. anginosus & out & yes & P. aeruginosa, S. aureus \\
BLEMI & CF & 20.09 .2012 & S. anginosus & in & yes & P. aeruginosa \\
LEXVL & CF & 04.10 .2012 & S. intermedius & out & yes & P. aeruginosa \\
LEVVE & CF & 09.10 .2012 & S. intermedius & out & yes & P. aeruginosa \\
POLRA & CF & 18.12 .2012 & S. anginosus & in & no & P. aeruginosa \\
HRAKA & CF & 15.01 .2013 & S. anginosus & out & no & P. aeruginosa \\
LEXVL & CF & 28.02 .2013 & S. anginosus & out & no & P. aeruginosa \\
CECNA & CF & 16.04 .2013 & S. anginosus & out & no & no \\
BLEMI & CF & 25.06 .2013 & S. anginosus & out & yes & P. aeruginosa, K. oxytoca \\
LEVVE & CF & 25.09 .2013 & S. intermedius & in & yes & P. aeruginosa \\
KOPJI & CF & 25.10 .2013 & S. anginosus & in & yes & P. aeruginosa \\
TESJA & CF & 07.11 .2013 & S. anginosus & out & yes & P. aeruginosa \\
LEXVL & CF & 22.11 .2013 & S. constellatus & out & yes & P. aeruginosa \\
HACJA & COPD & 15.11 .2012 & S. anginosus & out & no & H. influenzae \\
SKAJA & COPD & 17.04 .2013 & S. constellatus & in & yes & no \\
LINDA & COPD & 27.05 .2013 & S. anginosus & out & yes & P. aeruginosa \\
PYTJI & COPD & 27.05 .2013 & S. constellatus & in & yes & no \\
VAGRO & COPD & 15.07 .2013 & S. anginosus & in & yes & no \\
VECDA & COPD & 09.07 .2013 & S. anginosus & in & no & no \\
VAGRO & COPD & 12.08 .2013 & S. anginosus & in & yes & no \\
LINDA & COPD & 07.11 .2013 & S. anginosus & out & yes & P. aeruginosa \\
ZEMJA & COPD & 25.11 .2013 & S. anginosus & in & yes & M. catarrhalis \\
HACJA & COPD & 11.12 .2013 & S. anginosus & out & no & no \\
\hline
\end{tabular}

+ inhaled colistin + oral clarithromycin, based on earlier finding of $P$. aeruginosa in sputum. The serum CRP level was 59.0 at admission and decreased to normal levels during therapy that resulted in remission. In case 2 , a 76-year-old man suffering from stage 3 COPD was admitted to hospital because of sudden dyspnoea. The serum CRP level was 3.6 only at admission and no other signs of bacterial etiology were found. Therefore the patient was treated symptomatically, then he recovered completely. In case 3 the same patient as in case 2 was admitted to hospital because of exacerbation 20 days after complete recovery from the previous exacerbation described as case 2. This time, serum CRP was 94.4 and decreased to normal levels during successful treatment by clarithromycin. In case 4, a 56-year-old men suffering from stage 4 COPD was admitted to hospital because of exacerbation and pneumonia. Serum CRP level was 171.9 at admission and decreased to normal levels during successful treatment by moxifloxacin.

\section{DISCUSSION}

\section{CF patients}

Our $17.6 \%$ recovery rate in samples from adult $\mathrm{CF}$ patients was lower than the $33.2 \%$ in the study at the Adult Cystic Fibrosis Unit, University of Calgary ${ }^{11}$, most probably because in our study, SMG had to be present in a sample at a concentration of at least $10^{6} \mathrm{CFU} / \mathrm{mL}$ to be successfully recovered. We decided to set this cut-off both for economic reasons (reduction of plate numbers) and because it is widely accepted for distinguishing po- tentially significant pathogens from colonisers ${ }^{17}$. Another study that used selective Mitis-Salivarius agar with 1\% tellurite to recover viridans group streptococci was able to recover SMG in $8.9 \%$ (4/45) of adult CF patients, all isolates being $S$. anginosus ${ }^{18}$. Because $\mathrm{SMG}$ have already been correlated with clinical data in CF patients with exacerbated disease and our data are in accordance with these findings, we did not analyse cases of SMG positive $\mathrm{CF}$ patients further. Interestingly, $P$. aeruginosa was always found in CF patients who both suffered exacerbation and were positive for SMG. In all of these cases, $P$. aeruginosa had colonized the patients prior to the recovery of SMG. Recently, SMG were demonstrated to increase both production of $P$. aeruginosa virulence factors in coculture and also its virulence in co-infection in the wax moth larvae (Galleria mellonella) infection mode ${ }^{19}$. Thus, it is conceivable that new acquisition or excessive proliferation of SMG can trigger exacerbation in CF patients by stimulating the expression of virulence factors in $P$. aeruginosa that hitherto colonised the lungs.

\section{COPD patients}

In addition to $\mathrm{CF}$ patients, we also recovered $\mathrm{SMG}$ from as much as $10.3 \%(n=9)$ of sputum samples from COPD patients $(n=87)$. The incidence of SMG in COPD has not been studied systematically to date. To our knowledge, SMG was reported to be recovered from 3 of 4 COPD cases and from 6 of 11 bronchiectasis cases so far, without evaluating the relationship to clinical status ${ }^{12}$. Further, they were also noted in two cases of idiopathic chronic bronchiectasis exacerbations ${ }^{9}$. Unfortunately, we found no difference between the frequencies of exacerba- 
tions in SMG positive versus negative patients and were thus unable to prove that SMG played any role in exacerbations. The small number of cases was the most probable cause because there was also no difference or no significant difference in the frequencies of such well-established respiratory pathogens as $H$. influenzae and $K$. pneumoniae, respectively. Therefore, we tried to shed more light on the relationship between SMG and COPD exacerbation by analysing individual cases. There were 4 cases of exacerbation where no other bacterial pathogen was detected at the time of SMG recovery. In one of these cases, the bacterial etiology of exacerbation was excluded based on very low CRP level and positive response to symptomatic therapy without administration of antibiotics. In three of these cases, high initial CRP levels were observed together with remission following empirical antibiotic therapy. All the antibiotics or combinations used in these cases had the potential to be active against SMG. Thus, in 3 of the 4 cases of COPD exacerbations where no bacterial pathogen other than SMG was isolated, the exacerbations were most probably of bacterial origin and the most probable causative agent was SMG. This is highly suggestive of an etiological role of SMG in exacerbations of COPD, despite the absence of statistical evidence. Similarly, the status of SMG as opportunist pathogens in CF does not rely on statistical evaluation but rather on analysis of cases where the clinical course supports the causative role of SMG in acute exacerbations ${ }^{8,9}$.

\section{Interspecies differences in virulence?}

Notably, S. intermedius or S. constellatus were recovered in 6 cases that were all classified as exacerbations (100\%), whereas S. anginosus was recovered in 17 cases, where only 10 were classified as exacerbations (59\%). Although this difference was not statistically significant, possibly because of the small sample ( $P=0.115$, Fisher's exact test), similar findings were statistically significant in a large collection of SMG isolates from cases of infection in different body sites, where $S$. intermedius or $S$. constellatus were more likely to cause deep abscess ( $86 \%$ and $73 \%$ of isolates of each species, respectively) than was $S$. anginosus $(19 \%)^{20}$. This indicates that the clinical relevance of $S$. anginosus may be lower than that of $S$. intermedius or constellatus in lower respiratory pathways as well. Interestingly, the assumed greater virulence of $S$. intermedius (SI) and constellatus (SC) than that of $S$. anginosus (SA) is also supported by the higher incidence of potential virulence factors observed in isolates included in this study. Namely, hyaluronidase activity was present in all SI and SC isolates as compared to its absence in all SA isolates, capsule was formed by all SI and SC isolates compared to $44 \%$ of SA isolates and any type of haemolysis was observed in $75 \%$ of SI and in all SC isolates, compared to $50 \%$ of SA isolates after prolonged cultivation on $\mathrm{MH}$ agar with 7\% horse blood (Table 4). In our study, we were unable to detect human-specific haemolysins, like the $S$. intermedius intermedialysin. However, because human blood-based agar plates are not used routinely and our study aimed to support better recovery of SMG in routine settings in future, we prefer to report on the extent of haemolysis on sheep and horse blood that are generally available in routine diagnostic laboratories.

\section{Potential for better diagnostic performance}

In routine diagnostics, SMG streptococci recovered from respiratory tract are possibly reported as potentially pathogenic in the case that they draw the attention of a microbiologist because of beta-haemolysis, typically around minute colonies ${ }^{21}$. Unfortunately, in contrast to prolonged cultivation on $\mathrm{MH}$ agar supplemented with $7 \%$ horse blood, only one of our isolates showed betahaemolysis on CBA after 24-48 hours cultivation, the others showed either alpha-haemolysis or none. In such cases, SMG are typically considered non-pathogenic streptococci in respiratory pathways and escape the attention in routine settings. One way of overcoming this obstacle is the use of semi-selective media as the McKay agar $^{9,11}$ or the newly developed NAS agar ${ }^{12}$ that will grow mainly streptococci. However, 2-3 colonial morphotypes per sample are typically recovered and are then needed to be identified, making the SMG detection more laborious. Fortunately, identification of streptococcal isolates can now be achieved rapidly and conveniently using the MALDI TOF mass spectrometry. Direct detection and identification in sputum samples using PCR is another alternative, which offers the possibility of faster performance than cultivation followed by MALDI TOF MS. However, to take full advantage of its potential to speed up the detection, SMG-specific PCR would have to be performed on a daily basis. Both of the PCR approaches that we have tested, proved to be specific and reliable on our SMG isolates, however, we have not tested their performance in clinical samples directly, where inhibition may occur. For economic and easy use in routine diagnostics, nested PCR may be preferred if species identification is desired and more costly probes are to be avoided. Also, the detection limit appears to be $10-100 \times$ lower in favour of nested PCR. On the other hand, two-round amplification is more labour-intensive and prone to false positivity if cross-contamination is not under strict control. Another disadvantage of PCR in general is the lack of SMG quantification in relation to the context of other bacterial flora. Such quantification on agar plates would support better decision-making on colonization versus infection status compared to sole PCR quantification.

\section{CONCLUSION}

The circumstantial evidence from our results and the results of others indicate that SMG streptococci may represent typical opportunist pathogens colonising the airways and occasionally causing infection, possibly with increased frequency in hosts suffering chronic lung disease. In such case, S. intermedius and S. constellatus would be more pathogenic in lower respiratory pathways than $S$. anginosus, as already demonstrated in other body locations. In our study, we newly demonstrate that SMG might have a role in exacerbations of COPD. Appropriate and accurate identification of streptococcal isolates in 
samples from lower respiratory pathways together with careful clinical interpretation of the results should be helpful in improving the management of a fraction of exacerbations in both CF and COPD. Conventional culture on selective media followed by MALDI TOF MS identification or specific PCR amplification should both be able to facilitate SMG differentiation. All of our isolates were uniformly susceptible to penicillin, which is in accordance with the $94.4 \%$ of isolates found susceptible in a study of 180 strains recovered from clinically significant samples ${ }^{22}$. This offers the patient an option of narrow-spectrum targeted therapy with few side effects after detecting SMG as the sole suspect pathogen, or the option of antipseudomonal treatment combined with anti-SMG targeted penicillin treatment for enhanced effect and faster recovery in cases where the antipseudomonal treatment does not include a beta-lactam antibiotic and SMG were detected along with $P$. aeruginosa.

Acknowledgment: Supported by Ministry of Health of the Czech Republic, grant nr. NT/13560.

Author contributions: LN: biochemical profiling, Lancefield classification, penicillin susceptibility testing, DNA extraction, PCR; JB: MALDI TOF MS species identification; RN: capsule formation; JZ, PJ, VK, MZ and FK: selection of patients, acquisition of informed consent, sampling, clinical data; PP: processing and primary cultivation; VR: project leader, selection of morphotypes, hyaluronidase assay, data management, manuscript preparation.

Conflict of interest statement: The authors state that there are no conflicts of interest regarding the publication of this article.

\section{REFERENCES}

1. Miller SD, Mauff AC, Koornhof HJ. Streptococcus milleri causing infection in man. S Afr Med J 1983;63:684-6.

2. Shinzato T, Saito A. The Streptococcus milleri group as a cause of pulmonary infections. Clin Infect Dis 1995;21 Suppl 3:S238-43.

3. Wong CA, Donald F, Macfarlane JT. Streptococcus milleri pulmonary disease: a review and clinical description of 25 patients. Thorax 1995;50:1093-6.

4. Okada F, Ono A, Ando Y, Nakayama T, Ishii H, Hiramatsu K, Sato H, Kira A, Otabe M, Mori H. High-resolution CT findings in Streptococcus milleri pulmonary infection. Clin Radiol 2013;68:e331-7.

5. Porta G, Rodriguez-Carballeira M, Gomez L, Salavert M, Freixas N, Xercavins M, Garau J. Thoracic infection caused by Streptococcus milleri. Eur Respir J 1998;12:357-62.

6. Kobashi Y, Mouri K, Yagi S, Obase Y, Oka M. Clinical analysis of cases of empyema due to Streptococcus milleri group. Jpn J Infect Dis 2008;61:484-6.
7. Lee EY, Khatwa U, McAdam AJ, Bastos M, Mahmood SA, Ervoes JP, Boiselle PM. Streptococcus milleri group pleuropulmonary infection in children: computed tomographic findings and clinical features. J Comput Assist Tomogr 2010;34:927-32.

8. Parkins MD, Sibley CD, Surette MG, Rabin HR. The Streptococcus milleri group--an unrecognized cause of disease in cystic fibrosis: a case series and literature review. Pediatr Pulmonol 2008;43:490-7.

9. Sibley CD, Parkins MD, Rabin HR, Duan K, Norgaard JC, Surette MG. A polymicrobial perspective of pulmonary infections exposes an enigmatic pathogen in cystic fibrosis patients. Proc Natl Acad Sci U S A 2008; 105:15070-5.

10. Cade A, Denton M, Brownlee KG, Todd N, Conway SP. Acute bronchopulmonary infection due to Streptococcus milleri in a child with cystic fibrosis. Arch Dis Child 1999;80:278-9.

11. Sibley CD, Grinwis ME, Field TR, Parkins MD, Norgaard JC, Gregson DB, Rabin HR, Surette MG. McKay agar enables routine quantification of the 'Streptococcus milleri' group in cystic fibrosis patients. J Med Microbiol 2010;59:534-40.

12. Waite RD, Wareham DW, Gardiner S, Whiley RA. A simple, semiselective medium for anaerobic isolation of anginosus group streptococci from patients with chronic lung disease. J Clin Microbiol 2012;50:1430-2.

13. Yamaura M, Sato T, Echigo S, Takahashi N. Quantification and detection of bacteria from postoperative maxillary cyst by polymerase chain reaction. Oral Microbiol Immunol 2005;20:333-8.

14. Olson AB, Sibley CD, Schmidt L, Wilcox MA, Surette MG, Corbett $C R$. Development of real-time PCR assays for detection of the Streptococcus milleri group from cystic fibrosis clinical specimens by targeting the cpn60 and 16S rRNA genes. J Clin Microbiol 2010;48:1150-60.

15. Desar IM, de Boer M, Bens CC, Jacobs JA, Mouton JW, Dofferhoff AS, Klaassen $\mathrm{CH}$. Rapid and reliable identification of Streptococcus anginosus group isolates to the species level by real-time PCR and melting curve analysis. J Microbiol Methods 2008;75:372-4.

16. Skalka B. [Hyaluronidase test in the diagnosis of staphylococci]. Vet Med (Praha) 1985;30:373-8.

17. Miravitlles M, Espinosa C, Fernandez-Laso E, Martos JA, Maldonado JA, Gallego M. Relationship between bacterial flora in sputum and functional impairment in patients with acute exacerbations of COPD. Study Group of Bacterial Infection in COPD. Chest 1999;116:40-6.

18. Maeda Y, Elborn JS, Parkins MD, Reihill J, Goldsmith CE, Coulter WA, Mason C, Millar BC, Dooley JS, Lowery CJ, Ennis M, Rendall JC, Moore JE. Population structure and characterization of viridans group streptococci (VGS) including Streptococcus pneumoniae isolated from adult patients with cystic fibrosis (CF). J Cyst Fibros 2011;10:133-9.

19. Whiley RA, Sheikh NP, Mushtaq N, Hagi-Pavli E, Personne Y, Javaid $\mathrm{D}$, Waite RD. Differential potentiation of the virulence of the Pseudomonas aeruginosa cystic fibrosis liverpool epidemic strain by oral commensal streptococci. J Infect Dis 2014;209:769-80.

20. Claridge JE, 3rd, Attorri S, Musher DM, Hebert J, Dunbar S. Streptococcus intermedius, Streptococcus constellatus, and Streptococcus anginosus ("Streptococcus milleri group") are of different clinical importance and are not equally associated with abscess. Clin Infect Dis 2001;32:1511-5.

21. Poole PM, Wilson G. Infection with minute-colony-forming betahaemolytic streptococci. J Clin Pathol 1976;29:740-5.

22. Limia A, Jimenez ML, Alarcon T, Lopez-Brea M. Five-year analysis of antimicrobial susceptibility of the Streptococcus milleri group. Eur J Clin Microbiol Infect Dis 1999;18:440-4. 